\title{
DISSIPATIVE ENTROPY AND GLOBAL SMOOTH SOLUTIONS FOR RADIATION HYDRODYNAMICS AND MAGNETOHYDRODYNAMICS
}

\author{
CHRISTIAN ROHDE AND WEN-AN YONG
}

\begin{abstract}
The equations of ideal radiation magnetohydrodynamics (RMHD) serve as a fundamental mathematical model in many astrophysical applications. It is well-known that radiation can have a damping effect on solutions of associated initial-boundary-value problems. In other words, singular solutions like shocks can be prohibited.

In this paper, we consider discrete-ordinate approximations of the RMHD-system for general equations of state. If the magnetic fields are absent (i.e., if we consider radiation hydrodynamics), we prove the existence of global-in-time classical solutions for the Cauchy problem in one space dimension under an appropriate smallness condition on the inital data. We also show that counterparts of the compressive shock waves for the full RHD case and counterparts of the slow and fast MHD shock waves for the full RMHD-system can have structures in the presence of radiation if the amplitude is sufficiently small. Moreover, a new entropy function for the RMHD-system is presented.
\end{abstract}

\section{INTRODUCTION}

Consider an inviscid, quasi-neutral plasma of infinite conductivity. If we take into account the energy exchange due to the absorption of radiation, the dynamics of the plasma is governed by the equations of ideal magnetohydrodynamics coupled to a family of radiation transport equations $[9,11]$ :

$$
\begin{aligned}
\rho_{t}+\operatorname{div}(\rho \mathbf{v}) & =0 \\
(\rho \mathbf{v})_{t}+\operatorname{div}(\rho \mathbf{v} \otimes \mathbf{v}+\mathcal{P}) & =0 \\
\mathbf{B}_{t}+\operatorname{div}\left(\mathbf{v B}^{T}-\mathbf{B v}^{T}\right) & =0 \\
(\rho \mathcal{E})_{t}+\operatorname{div}(\rho \mathcal{E} \mathbf{v}+\mathcal{P} \mathbf{v}) & =\rho \oint_{\mathcal{S}^{2}}(I(x, t, \boldsymbol{\omega})-b(\theta)) d \boldsymbol{\omega} \\
I_{t}+c \boldsymbol{\omega} \cdot \nabla I & =c \rho(b(\theta)-I) .
\end{aligned}
$$

Here the unknowns are the density function of the fluid $\rho=\rho(\mathbf{x}, t)>0$, the velocity $\mathbf{v}=$ $\mathbf{v}(\mathbf{x}, t) \in \mathbb{R}^{3}$, the magnetic field $\mathbf{B}=\mathbf{B}(\mathbf{x}, t) \in \mathbb{R}^{3}$, the temperature $\theta=\theta(\mathbf{x}, t)>0$, and the radiation intensity $I=I(\mathbf{x}, t, \boldsymbol{\omega}) \geq 0$ for $(\mathbf{x}, t) \in \mathbb{R}^{3} \times(0, \infty)$ and $\boldsymbol{\omega} \in \mathcal{S}^{2}$. The subscript $t$ denotes the partial derivative with respect to the time variable $t$, while div and $\nabla$ are the usual divergence and gradient operators with respect to the spatial variable $\mathbf{x}$. $\otimes$ stands for the tensor product, the superscript $T$ denotes the transpose of vectors (or matrices), and the dot "." between two vectors means the scalar product. $c$ is the speed of light. The

Key words and phrases. Radiative MHD, entropy functions, dissipation, shock structures. 
generalized pressure tensor $\mathcal{P}$ is given by

$$
\begin{aligned}
\mathcal{P} & :=p \mathcal{I}_{3}+\mathcal{P}_{\text {mag }}, \\
\mathcal{P}_{\text {mag }} & :=\frac{1}{8 \pi}|\mathbf{B}|^{2} \mathcal{I}_{3}-\frac{1}{4 \pi} \mathbf{B B}^{T}
\end{aligned}
$$

with $\mathcal{I}_{3}$ being the unit matrix in $\mathbb{R}^{3 \times 3}$;

$$
\mathcal{E}=e+\frac{1}{8 \pi \rho}|\mathbf{B}|^{2}, \quad e=\varepsilon+\frac{1}{2}|v|^{2} ;
$$

while $p=p(\rho, \theta), b=b(\theta)$ and $\varepsilon=\varepsilon(\rho, \theta)$ are three given smooth functions denoting the hydrodynamical pressure, the Planck function and the specific energy, respectively.

Throughout this paper, we make the standard thermodynamical assumptions (cf. [3])

$$
\theta, p_{\rho}(\rho, \theta), \varepsilon_{\theta}(\rho, \theta), p_{\theta}(\rho, \theta)>0 .
$$

Recall that the specific entropy $s=s(\rho, \varepsilon)$ is determined through the Gibbs relation

$$
\theta d s=d \varepsilon+p d \tau, \quad \tau:=1 / \rho,
$$

from which we can easily get the following formula

$$
p=\theta p_{\theta}+\rho^{2} \varepsilon_{\rho} .
$$

For the Planck function $b=b(\theta)$, we assume that

$$
b(\theta)>0, \quad b^{\prime}(\theta)>0
$$

for all $\theta$ under consideration.

On the physical grounds, it is usually assumed that the initial magnetic field $\mathbf{B}(\mathbf{x}, 0)$ is solenoidal. In this case, the $\mathbf{B}$-equation in (1.1) implies that the possible solution satisfies

$$
\operatorname{div} \mathbf{B}(\cdot, t)=0 \quad \forall t>0 .
$$

This, together with the thermodynamical assumptions (1.4), ensures that $-\rho s(\rho, \varepsilon)$ is a strictly convex entropy function for the homogeneous MHD system

$$
\begin{aligned}
\rho_{t}+\operatorname{div}(\rho \mathbf{v}) & =0 \\
(\rho \mathbf{v})_{t}+\operatorname{div}(\rho \mathbf{v} \otimes \mathbf{v}+\mathcal{P}) & =0 \\
\mathbf{B}_{t}+\operatorname{div}\left(\mathbf{v} \mathbf{B}^{T}-\mathbf{B v}^{T}\right) & =0 \\
(\rho \mathcal{E})_{t}+\operatorname{div}(\rho \mathcal{E} \mathbf{v}+\mathcal{P} \mathbf{v}) & =0
\end{aligned}
$$

By using such an entropy function for $(1.8)$, it was proved in $[12,17]$ that the initial-value problem for (1.1) has a local-in-time classical solution. Moreover, it was shown in [17] that for large enough initial data, the classical solutions cannot persist for all times. On the other hand, it was indicated in the astrophysical literature (cf. the rather old work [5] or the more recent contributions [1]) that system (1.1) can have smooth traveling wave solutions if the far-field states are sufficiently close together. However, except $[6,7,8]$, these merely numerical observations have not been verified rigorously up to our knowledge. We believe that these phenomena are due to the dissipative effects of radiation.

The goal of this paper is to analyse dissipative effects of radiation at a rigorous level. The main results will be presented for a class of discrete-ordinate approximations of (1.1). Such discrete-ordinate approximations are of the form of hyperbolic systems of balance 
laws. By introducing a new disspative entropy function, we show that the discrete-ordinate approximations possess the stability structure proposed in [15] for general hyperbolic systems of balance laws. Moreover, the Kawashima condition [13] is examined for the discreteordinate approximations. With these discussions, we use the existence theories developed in $[15,16,2]$ to obtain the following conclusions:

(A): The Cauchy problem for one-dimensional discrete-ordinate RHD-systems (i.e., (1.1) with $\mathbf{B} \equiv 0$ ) has a unique smooth solution, provided that the initial data are close to a constant equilibrium state in a certain Sobolev space (see Theorem 5.3).

(B): Both the discrete ordinate RHD- and RMHD-systems have smooth planar solutions of traveling wave type, if one of the two asymptotic states is static and the two are close to each other (see Theorem 6.2 for the RHD case and Theorem 7.2 for the RMHD case).

About these results, we comment as follows. The discrete-ordinate approximations can be regarded as approximate models for radiation (magneto)hydrodynamics and are often used in practical numerical simulations [1]. The new dissipative entropy function is interesting on its own and is an essential tool in the subsequent discussions. The result in (A) do not apply to the multi-dimensional case and not to the MHD-system, since the Kawashima condition does not hold here. This, however, implies by no means that radiation does not prohibit the development of singularities in multiple space dimensions. The results in (B) can be interpreted in the sense that compressive shock waves of the Euler equations or the equations of ideal magnetohyrodynamics can have structure in the presence of radiation. They are analoguous to those on the existence of viscous profiles for the same kind of shock waves (cf. Part IV of [14]). Moreover, the results in (B) can be viewed as applications of the existence theory developed in [2], which does not contain any applications. In fact, the abstract result in $[2]$ requires a technical condition and this condition has not been verified for any specific problems before.

This paper is organized as follows. In Sect. 2 we review the existence theories in $[15,16,2]$. The discrete-ordinate approximations will be presented in Sect. 3. In Sect. 4 we introduce a new dissipative entropy function for the discrete-ordinate approximations. Sect. 5 is devoted to the Cauchy problem for the one-dimensional RHD-system. In Sect. 6 we address the question of existence of smooth planar solutions of traveling wave type for the discrete ordinate RHD- and RMHD-systems.

\section{Existence Theories for Hyperbolic Balance Laws}

In this section, we review the existence theories developed in $[2,15,16]$ for general hyperbolic systems of balance laws:

$$
U_{t}+\sum_{j=1}^{d} F_{j}(U)_{x_{j}}=Q(U), \quad \text { in } \mathbb{R}^{d} \times(0, \infty),
$$

subject to the initial condition

$$
U(\mathbf{x}, 0)=U_{0}(\mathbf{x}) .
$$

Here $\mathbf{x}=\left(x_{1}, x_{2}, \cdots, x_{d}\right)$ and the unknown $U=U(\mathbf{x}, t): \mathbb{R}^{d} \times[0, \infty) \rightarrow \mathcal{U}$ takes values in an open set $\mathcal{U} \subset \mathbb{R}^{n}$. We assume, throughout this section, that $F_{1}, \ldots, F_{d}, Q \in C^{\infty}\left(\mathcal{U}, \mathbb{R}^{n}\right)$. More assumptions on $U_{0}: \mathbb{R}^{d} \rightarrow \mathcal{U}$ will be specified below. In what follows, we denote by 
$0_{l \times m}$ a matrix of dimension $l \times m$, which has only zeros as entries and by $\mathcal{I}_{l}$ the identity matrix of dimension $l$.

As is well-known, global solutions of the initial-value problem (2.1) with (2.2) can contain discontinuities like shock and contact waves. Under appropriate structural conditions on (2.1), the formation of discontinuities can be prevented if the initial data are close to a constant state $\bar{U}$ satisfying $Q(\bar{U})=0$. A general result in this aspect is

Theorem 2.1. $\left[15\right.$, Theorem 3.1] Let $U_{0} \in H^{s}\left(\mathbb{R}^{d}\right)$ with $s \geq[d / 2]+2$ an integer and $\bar{U} \in \mathcal{U}$ be such that $Q(\bar{U})=0$. Suppose the following conditions are satisfied.

(i) There exists a positive integer $r \leq n$, two constant matrices $E_{1} \in \mathbb{R}^{(n-r) \times n}$ and $E_{2} \in \mathbb{R}^{r \times n}$ such that $E_{1} Q(U)=0$ for all $U$, and both

$$
E=\left(\begin{array}{c}
E_{1} \\
E_{2}
\end{array}\right) \in \mathbb{R}^{n \times n} \quad \text { and } \quad E_{2} Q_{U}(\bar{U}) E^{-1}\left(\begin{array}{c}
0_{(n-r) \times r} \\
\mathcal{I}_{r}
\end{array}\right) \in \mathbb{R}^{r \times r}
$$

are invertible,

(ii) there is a strictly convex function $\eta \in C^{2}(\mathcal{U}, \mathbb{R})$ and a function $\Psi_{j} \in C^{2}(\mathcal{U}, \mathbb{R})$ such that

$$
\eta_{U}(U)^{T} F_{j U}(U)=\Psi_{j U}(U)^{T}, \quad j=1, \ldots, d,
$$

holds for all $U \in \mathcal{U}$,

(iii) for a certain neighborhood $G \subset \subset \mathcal{U}$ of $\bar{U}$, there is a constant $c_{G}$ such that

$$
\left(\eta_{U}(U)-\eta_{U}(\bar{U})\right) \cdot Q(U) \leq-c_{G}|Q(U)|^{2}
$$

holds for all $U \in G$,

(iv) the kernel of the Jacobian $Q_{U}(\bar{U})$ contains no eigenvectors of the matrices

$$
\sum_{j=1}^{d} \xi_{j} F_{j U}(\bar{U}) \quad\left(\boldsymbol{\xi} \in \mathcal{S}^{d-1}\right) .
$$

Then there exists a constant $C>0$ such that for any initial data $U_{0}(\mathbf{x})$ satisfying

$$
\left\|U_{0}-\bar{U}\right\|_{H^{s}(\mathbb{R})} \leq C,
$$

the initial-value problem (2.1) with (2.2) has a unique global solution $U=U(\mathbf{x}, t)$ satisfying $U-\bar{U} \in C\left([0, \infty) ; H^{s}\left(\mathbb{R}^{d}\right)\right)$.

Remark 2.1. (1). With the matrix $E$ from condition (i) above, we define

$$
\left(\begin{array}{l}
u \\
v
\end{array}\right)=E U, \quad q(u, v)=E_{2} Q(U)=E_{2} Q\left(E^{-1}\left(\begin{array}{l}
u \\
v
\end{array}\right)\right) .
$$

Then

$$
q_{v}(u, v)=E_{2} Q_{U}(U) E^{-1}\left(\begin{array}{c}
0_{(n-r) \times r} \\
\mathcal{I}_{r}
\end{array}\right)
$$

is invertible at $\bar{U}$. This is the requirement of Theorem 3.1 in [15].

(2). Condition (iv) of Theorem 2.1 is usually referred to as Kawashima's condition. It is a convenient condition in many applications. However, this condition seems not a necessary, but just a sufficient, condition for the global existence result. In fact, it will fail to hold true for the multidimensional RMHD-system as we will show below. Nevertheless, we believe that the Cauchy problem for the RMHD system still have 
globally defined smooth solutions with initial data close to a constant equilibrium state.

Now let $\bar{U}$ be a constant equilibrium state as in Theorem 2.1. Condition (i) implies in particular that $E_{2} U$ can be expressed in terms of $E_{1} U$, say,

$$
E_{2} U=h\left(E_{1} U\right)
$$

with $h=h(u)$ a certain given $(n-r)$-vector valued function of $u$. Thus, as in Remark 2.1 we set $u=E_{1} U$ and $v=E_{2} U$. In particular, we have

$$
\bar{v}:=E_{2} \bar{U}=h\left(E_{1} \bar{U}\right)=h(\bar{u}) .
$$

Then we call

$$
u_{t}+\sum_{j=1}^{d} f_{j}(u)_{x_{j}}=0 \text { in } \mathbb{R}^{d} \times(0, \infty)
$$

with

$$
f_{j}(u)=E_{1} F_{j}\left(M^{-1}\left(\begin{array}{c}
u \\
h(u)
\end{array}\right)\right)
$$

the equilibrium system associated to (2.1). By analysing the (possibly simpler) equilibrium systems, one can prove the existence of traveling-wave solutions for (2.1). More precisely, we have

Theorem 2.2. [16, 2] Under conditions (i), (ii) and (iii) of Theorem 2.1, fix $\bar{U}$ and $\boldsymbol{\xi} \in \mathcal{S}^{d-1}$, let $\lambda$ be an isolated eigenvalue of the characteristic matrix

$$
\sum_{j} \xi_{j} f_{j u}(\bar{u}), \quad \bar{u}=E_{1} \bar{U} .
$$

Assume that the piecewise constant function

$$
u(\mathbf{x}, t)=\left\{\begin{array}{l}
\bar{u}, \quad \sum_{j} \xi_{j} x_{j} \leq s_{*} t \\
u_{+}, \quad \sum_{j} \xi_{j} x_{j} \geq s_{*} t
\end{array}\right.
$$

is a weak solution for the equilibrium system (2.4) with $s_{*}=s_{*}\left(\bar{u}, u_{+}\right)<\lambda=\lim _{u \rightarrow \bar{u}} s_{*}\left(u_{-}, u\right)$. Then the following holds true.

(a) If $\lambda$ is not an eigenvalue of the characteristic matrix $\sum_{j} \xi_{j} F_{j U}(\bar{U})$, then for any $u_{+}$ close to $\bar{u}$, the system (2.1) has a unique classical solution of the form

$$
U(\mathbf{x}, t)=\phi\left(\sum_{j} \xi_{j} x_{j}-s_{*} t\right),
$$

where $\phi \in C^{1}(\mathbb{R})$ satisfies $\phi(-\infty)=\bar{U}$ and $\phi(+\infty)=E^{-1}\left(u_{+}, h\left(u_{+}\right)\right)^{T}$.

(b) In case $\lambda$ is an eigenvalue of $\sum_{j} \xi_{j} F_{j U}(\bar{U})$, if $\lambda$ is isolated also for $\sum_{j} \xi_{j} F_{j U}(\bar{U})$ and

$$
\operatorname{Ker}\left(Q_{U}(\bar{U})\right) \cap \operatorname{Ker}\left(\sum_{j=1}^{d} \xi_{j} F_{j U}(\bar{U})-\lambda \mathcal{I}_{n}\right)=\{0\},
$$

then (2.1) still has a smooth solution of the above form. 
Remark 2.2. As was pointed out in [2], the assumption that $\lambda$ is isolated for $\sum_{j} \xi_{j} F_{j U}(\bar{U})$ can be relaxed to be that the eigenvalues $\Lambda(U)$ of $\sum_{j=1}^{d} \xi_{j} F_{j U}(U)$ tending to $\lambda$ as $U$ goes to $\bar{U}$ are identical for all $U$ close to $\bar{U}$.

\section{Discrete-Ordinate Approximations}

In this section, we present two model systems derived from (1.1), which can be viewed as an system of infinitely many balance laws. Note that the energy equation in (1.1) has an integral-type source term. In practical computations $[11,1]$, the integral is often replaced with a quadrature formula, i.e., a finite sum of the form

$$
\sum_{l=1}^{L} c_{l}\left(I\left(\mathbf{x}, t, \boldsymbol{\omega}_{l}\right)-b(\theta)\right)
$$

with $c_{l}>0$ and $\boldsymbol{\omega}_{l} \in \mathcal{S}^{2}$.

For the sake of simplicity, we assume that all $c_{l}$ are equal, which corresponds to an equalsized partition of the sphere. About such partitions, we remark as follows.

Remark 3.1. For our purposes, it does not matter how the discrete ordinates $\boldsymbol{\omega}_{l}$ are chosen. However, for concrete numerical schemes, it might be desirable to start from an equal-sized partition of the sphere and to take the directions $\boldsymbol{\omega}_{l}$ as vectors through the middel points of the single surface elements. For such constructions, we refer to [10].

Substituting the integral in (1.1) with the finite sum and setting $I_{l}=c_{l} I\left(\mathbf{x}, t, \boldsymbol{\omega}_{l}\right)$, we obtain the following discrete-ordinate system of $(8+L)$ equations:

$$
\begin{aligned}
\rho_{t}+\operatorname{div}(\rho \mathbf{v}) & =0 \\
(\rho \mathbf{v})_{t}+\operatorname{div}(\rho \mathbf{v} \otimes \mathbf{v}+\mathcal{P}) & =0 \\
\mathbf{B}_{t}+\operatorname{div}\left(\mathbf{v B}^{T}-\mathbf{B v}^{T}\right) & =0 \\
(\rho \mathcal{E})_{t}+\operatorname{div}(\rho \mathcal{E} \mathbf{v}+\mathcal{P} \mathbf{v}) & =\rho \sum_{l=1}^{L}\left(I_{l}-b(\theta)\right) \\
I_{l t}+c \boldsymbol{\omega}_{l} \cdot \nabla I_{l} & =c \rho\left(b(\theta)-I_{l}\right), \quad l=1, \ldots, L,
\end{aligned}
$$

together with the divergence-free constraint in (1.7): $\operatorname{div} \mathbf{B}(\mathbf{x}, t)=0$.

We are interested in planar solutions of (3.1). Without loss of generality, we assume that all quantities are independent of the $\left(x_{2}, x_{3}\right)$-variables. Note that then the divergence-free constraint leads to $B_{1}(\mathbf{x}, t)=$ const. If we assume $B_{1}(\mathbf{x}, t) \neq 0$ and rescale the transversal velocity field $\mathbf{w}:=\left(v_{2}, v_{3}\right)^{T}$ and the transversal magnetic field $\mathbf{c}:=\left(B_{2}, B_{3}\right)^{T}$ appropriately, we get with $x=x_{1}$ the system

$$
\begin{aligned}
\rho_{t}+(\rho v)_{x} & =0 \\
(\rho v)_{t}+\left(\rho v^{2}+p+|\mathbf{c}|^{2} / 2\right)_{x} & =0 \\
(\rho \mathbf{w})_{t}+(\rho v \mathbf{w}-\mathbf{c})_{x} & =0 \\
\mathbf{c}_{t}+(v \mathbf{c}-\mathbf{w})_{x} & =0 \\
(\rho \mathcal{E})_{t}+\left(\rho \mathcal{E} v+v p+v|\mathbf{c}|^{2} / 2-\mathbf{w} \cdot \mathbf{c}\right)_{x} & =\rho \sum_{l=1}^{L}\left(I_{l}-b(\theta)\right), \\
I_{l t}+c \omega_{l} I_{l x} & =c \rho\left(b(\theta)-I_{l}\right), \quad l=1, \ldots, L .
\end{aligned}
$$


Note that we have used $v:=v_{1}$ for the longitudinal velocity and $\omega_{l}:=\boldsymbol{\omega}_{l, 1}$. This system is closed by

$$
\mathcal{E}=\varepsilon+\frac{1}{2}\left(v^{2}+|\mathbf{w}|^{2}\right)+\frac{1}{2 \rho}|\mathbf{c}|^{2} .
$$

We also consider the case with vanishing magnetic fields, which leads to the radiative hydrodynamical (RHD-) system

$$
\begin{aligned}
\rho_{t}+\operatorname{div}(\rho \mathbf{v}) & =0, \\
(\rho \mathbf{v})_{t}+\operatorname{div}\left(\rho \mathbf{v} \otimes \mathbf{v}+p \mathcal{I}_{3}\right) & =0, \\
(\rho e)_{t}+\operatorname{div}(\rho e \mathbf{v}+p \mathbf{v}) & =\rho \sum_{l=1}^{L}\left(I_{l}-b(\theta)\right), \\
I_{l t}+c \boldsymbol{\omega}_{l} \cdot \nabla I_{l} & =c \rho\left(b(\theta)-I_{l}\right), \quad l=1, \ldots, L,
\end{aligned}
$$

with

$$
e=\varepsilon+\frac{1}{2}|\mathbf{v}|^{2}
$$

\section{Entropy Structure of the Discrete-Ordinate Approximations}

In this section, we show that the discrete-ordinate systems (3.2) and (3.4), as systems of balance laws, admit the conditions (i)-(iii) in Theorem 2.1. In particular, we will introduce a new entropy function for (3.2) and (3.4).

4.1. The RHD-System. The discrete-ordinate model (3.4) is of the form (2.1) with $n=$ $5+L$ and

$$
U=\left(\begin{array}{c}
\rho \\
\rho v_{1} \\
\rho v_{2} \\
\rho v_{3} \\
\rho e \\
I_{1} \\
\vdots \\
I_{L}
\end{array}\right), \quad F_{j}(U)=\left(\begin{array}{c}
\rho v_{j} \\
\rho v_{1} v_{j}+\delta_{1 j} p \\
\rho v_{2} v_{j}+\delta_{2 j} p \\
\rho v_{3} v_{j}+\delta_{3 j} p \\
\rho e v_{j}+p v_{j} \\
c \omega_{1 j} I_{1} \\
\vdots \\
c \omega_{L j} I_{L}
\end{array}\right), \quad Q(U)=\left(\begin{array}{c}
0 \\
0 \\
0 \\
0 \\
\rho \sum_{l=1}^{L}\left(I_{l}-b(\theta)\right) \\
-c \rho\left(I_{1}-b(\theta)\right) \\
\vdots \\
-c \rho\left(I_{L}-b(\theta)\right)
\end{array}\right)
$$

Here $\delta_{i j}$ is the standard Kronecker symbol. For this system, the state space is

$$
\mathcal{U}:=\left\{U \in(0, \infty) \times \mathbb{R}^{3} \times(0, \infty)^{L+1} \mid \theta \geq \theta_{0}\right\}
$$

with $\theta_{0}$ a given positive constant.

Thanks to the strict monotonicity of $b=b(\theta)$ assumed in (1.6), $b=b(\theta)$ has an inverse function $b_{*}=b_{*}(y)$, that is,

$$
\theta=b_{*}(b(\theta)), \quad \forall \theta \geq \theta_{0} .
$$

Note that $b_{*}=b_{*}(y)$ is also strictly increasing. Let $s=s(\rho, \varepsilon)$ be the specific entropy introduced in (1.5). Define

$$
\eta(U)=-\rho s(\rho, \varepsilon)-\sum_{l=1}^{L} \int_{b\left(\theta_{0}\right)}^{I_{l}} \frac{d y}{c b_{*}(y)} .
$$


For later use, we compute

$$
\eta_{\rho e}(U)=-\frac{1}{\theta}, \quad \eta_{I_{l}}(U)=-\frac{1}{c b_{*}\left(I_{l}\right)}
$$

With $\eta=\eta(U)$ defined above, we turn to verify the conditions (i)-(iii) in Theorem 2.1.

Proposition 4.1. The function $\eta: \mathcal{U} \rightarrow \mathbb{R}$ defined in (4.2) is strictly convex. The functions $\Psi_{j}: \mathcal{U} \rightarrow \mathbb{R}$ defined by

$$
\Psi_{j}(U)=-\rho s(\rho, \varepsilon) v_{j}-\sum_{l=1}^{L} \omega_{l, j} \int_{b\left(\theta_{0}\right)}^{I_{l}} \frac{d y}{c b_{*}(y)} .
$$

satisfy

$$
\eta_{U}(U)^{T} F_{j U}(U)=\Psi_{j U}(U)^{T} \quad(j=1, \ldots, 3)
$$

for all $U \in \mathcal{U}$. Moreover, for any constant equilibrium state $\bar{U}$ and its any neighborhood $G \subset \subset \mathcal{U}$, there is a constant $c_{G}$ such that

$$
\left(\eta_{U}(U)-\eta_{U}(\bar{U})\right) \cdot Q(U) \leq-c_{G}|Q(U)|^{2}
$$

holds for all $U \in G$.

Proof: To see the strict convexity, it suffices to show that the two summands in (4.2) are both strictly convex with respect to the hydrodynamical variables and the radiation intensities, respectivley. Note that $\eta=\eta(U)$ contains no cross terms of the hydrodynamical variables and the radiation intensities. It is well-known (see, e.g., [3]) that the strict convexity of the first summand with respect to the hydrodynamical variables follows from the standard thermodynamical assumption in (1.4). Moreover, we deduce from (4.3) that

$$
\frac{\partial^{2}}{\partial I_{l} \partial I_{k}} \eta(U)=\delta_{k l} \frac{b_{*}^{\prime}\left(I_{l}\right)}{c b_{*}\left(I_{l}\right)^{2}}
$$

Thus, the convexity of the second summand is obvious due to (1.6). The verification of (4.4) is straightforward.

To show (4.5), we use (4.3) and the expression of $Q$ in (4.1) to compute

$$
\begin{aligned}
\eta_{U}(\bar{U}) \cdot Q(U) & =-\frac{\rho}{\bar{\theta}} \sum_{l=1}^{L}\left(I_{l}-b(\theta)\right)-\rho \sum_{l=1}^{L}\left(b(\theta)-I_{l}\right) \frac{1}{b_{*}\left(\bar{I}_{l}\right)} \\
& =\rho \sum_{l=1}^{L}\left(\left(I_{l}-b(\theta)\right) \frac{\bar{\theta}-b_{*}\left(\bar{I}_{l}\right)}{\bar{\theta} b_{*}\left(\bar{I}_{l}\right)}\right)=0 .
\end{aligned}
$$

Here we have used the components $\bar{I}_{l}$ of $\bar{U}$ satisfy $\bar{I}_{l}=b(\bar{\theta})$. Moreover, we compute

$$
\begin{aligned}
\eta_{U}(U) \cdot Q(U) & =-\frac{\rho}{\theta} \sum_{l=1}^{L}\left(I_{l}-b(\theta)\right)-\rho \sum_{l=1}^{L}\left(b(\theta)-I_{l}\right) \frac{1}{b_{*}\left(I_{l}\right)} \\
& =\rho \sum_{l=1}^{L}\left(\left(I_{l}-b(\theta)\right) \frac{\theta-b_{*}\left(I_{l}\right)}{\theta b_{*}\left(I_{l}\right)}\right) \\
& =-\rho \sum_{l=1}^{L}\left(\left(b(\theta)-I_{l}\right) \frac{b_{*}(b(\theta))-b_{*}\left(I_{l}\right)}{\theta b_{*}\left(I_{l}\right)}\right) .
\end{aligned}
$$


Since $b_{*}$ is a smooth and strictly increasing function, it follows from the mean-value theorem that $D_{l}\left(b(\theta), I_{l}\right):=\frac{b_{*}(b(\theta))-b_{*}\left(I_{l}\right)}{b(\theta)-I_{l}}>0$. Thus, we obtain

$$
\eta_{U}(U) \cdot Q(U)=-\frac{\rho}{\theta} \sum_{l=1}^{L} \frac{D_{l}\left(b(\theta), I_{l}\right)}{b_{*}\left(I_{l}\right)}\left(I_{l}-b(\theta)\right)^{2} .
$$

Now the inequality in (4.5) follows from the compactness of $G$ and the fact that $Q$ is a linear combination of the $\rho\left(I_{l}-b(\theta)\right)$ 's. This completes the proof.

We conclude this subsection with the following interesting observation.

Remark 4.1. From the expression of $Q$ in (4.1) and that of $\eta$ in (4.2), it is not difficult to see that

$$
Q(U)=-\mathcal{L}(U) \eta_{U}(U)
$$

holds with

$$
\mathcal{L}(U)=\rho\left(\begin{array}{ccccccc}
0_{4 \times 4} & 0_{4 \times 1} & 0_{4 \times 1} & 0_{4 \times 1} & 0_{4 \times 1} & \cdots & 0_{4 \times 1} \\
0_{1 \times 4} & \sum_{l} \sigma_{l} & -c \sigma_{1} & -c \sigma_{2} & -c \sigma_{3} & \cdots & -c \sigma_{L} \\
0_{1 \times 4} & -c \sigma_{1} & c^{2} \sigma_{1} & 0 & 0 & \cdots & 0 \\
0_{1 \times 4} & -c \sigma_{2} & 0 & c^{2} \sigma_{2} & 0 & \cdots & 0 \\
0_{1 \times 4} & -c \sigma_{3} & 0 & 0 & c^{2} \sigma_{3} & \cdots & 0 \\
\vdots & \vdots & \vdots & \vdots & \vdots & \vdots & \vdots \\
\vdots & \vdots & \vdots & \vdots & \vdots & \vdots & \vdots \\
0_{1 \times 4} & -c \sigma_{L} & 0 & 0 & 0 & \cdots & c^{2} \sigma_{L}
\end{array}\right)
$$

Here

$$
\sigma_{l}:=\frac{I_{l}-b(\theta)}{\theta^{-1}-b_{*}^{-1}\left(I_{l}\right)} .
$$

Since $\sigma_{l}>0$ for all $l$ due to (1.6), the matrix $\mathcal{L}(U)$ is symmetric and non-negative for all $U \in \mathcal{U}$. Moreover, its null space is

$$
\operatorname{span}\left\{e_{1}, e_{2}, \ldots e_{4}, e_{5}+c^{-1} \sum_{l \geq 6} e_{l}\right\}
$$

which is independent of $U$. Here $e_{k}$ is the $k^{\text {th }}$ column of the unit matrix $I_{(L+5)}$.

The above characterization of $Q=Q(U)$ means that the discrete-ordinate system (3.4) obeys a nonlinear variant of the Onsager reciprocal relation in non-equilibrium thermodynamics [4]. Here $Q=Q(U)$ is regarded as the thermodynamical flux, while the entropy variable $\eta_{U}(U)$ is the thermodynamical force. Through such a relation, the discrete-ordinate system (3.4) relates the irreversible processes directly to the entropy change $\eta_{U}(U)$.

4.2. The RMHD-System. The discussion in the previous subsection does not apply to the discrete-ordinate system (3.1) in multiple space dimensions, due essentially to the divergencefree constraint (1.7). However, it applies to the one-dimensional system (3.2). Indeed, (3.2) 
is of the form (2.1) with

$$
U=\left(\begin{array}{c}
\rho \\
\rho v \\
\rho \mathbf{w} \\
\mathbf{c} \\
\rho \mathcal{E} \\
I_{1} \\
\vdots \\
I_{L}
\end{array}\right), \quad F_{1}(U)=\left(\begin{array}{c}
\rho v \\
\rho v^{2}+p+|\mathbf{c}|^{2} / 2 \\
\rho v \mathbf{w}-\mathbf{c} \\
v \mathbf{c}-\mathbf{w} \\
\rho \mathcal{E} v+p v+v|\mathbf{c}|^{2} / 2-\mathbf{w} \cdot \mathbf{c} \\
c \omega_{1} I_{1} \\
\vdots \\
c \omega_{L} I_{L}
\end{array}\right), \quad Q(U)=\left(\begin{array}{c}
0 \\
0 \\
0 \\
0 \\
\rho \sum_{l=1}^{L}\left(I_{l}-b(\theta)\right) \\
-c \rho\left(I_{1}-b(\theta)\right) \\
\vdots \\
-c \rho\left(I_{L}-b(\theta)\right)
\end{array}\right) .
$$

Here the state space is

$$
\mathcal{U}:=\left\{U \in(0, \infty) \times \mathbf{R}^{5} \times(0, \infty)^{L+1} \mid \theta>\theta_{0}\right\},
$$

with some constant $\theta_{0}>0$.

Define $\eta=\eta(U)$ as in (4.2). Note that here the energy law is (3.3). The one-dimensional RMHD system (3.2) has the same structure as the (one-dimensional version of the) RHDsystem (3.4) and the coupling between the magnetohydrodynamical and the radiation part is weak. As was mentioned in the introduction, it is well-known that the function $-\rho s(\rho, \varepsilon)$ constitutes a strictly convex entropy function for the MHD system (1.8). Thus, the following result can be verified in the exactly same fashion as that for Proposition 4.1. Namely, conditions (i)-(iii) in Theorem 2.1 holds true for the one-dimensional system (3.2).

Proposition 4.2. The function $\eta: \mathcal{U} \rightarrow \mathbb{R}$ defined in (4.2) is strictly convex. The function $\Psi_{1}: \mathcal{U} \rightarrow \mathbb{R}$ defined with

$$
\Psi_{1}(U)=-\rho s(\rho, \varepsilon) v-\sum_{l=1}^{L} \int_{b\left(\theta_{0}\right)}^{I_{l}} \frac{d y}{c b_{*}(y)} \omega_{l} .
$$

satisfies

$$
\eta_{U}(U)^{T} F_{1 U}(U)=\Psi_{1 U}(U)^{T},
$$

for all $U \in \mathcal{U}$. Moreover, for any constant equilibrium state $\bar{U}$ and its any neighborhood $G \subset \subset \mathcal{U}$, there is a constant $c_{G}$ such that

$$
\left(\eta_{U}(U)-\eta_{U}(\bar{U})\right) \cdot Q(U) \leq-c_{G}|Q(U)|^{2}
$$

holds for all $U \in G$.

Finally, the interested reader is advised to verify Remark 4.1 for the one-dimensional discrete-ordinate system (3.2).

\section{Global Classical Solutions for One-Dimensional RhD Systems}

In this section, we present a result on the existence of global classical solutions to the Cauchy problem for the one-dimensional version of (3.4).

To this end, we check the conditions of Theorem 2.1. Let $\bar{U}$ be a given constant state satisfying $Q(\bar{U})=0$. Then it is obvious (cf. (4.1)) that

$$
\bar{I}_{l}=b(\bar{\theta}), \quad \forall l,
$$


with $\bar{\theta}$ the temperature corresponding to $\bar{U}$. Condition (i) is easily seen to be satisfied with $r=L$ and

$$
E_{1}=\left(\begin{array}{cc}
\mathcal{I}_{d+1} & 0_{(d+1) \times(L+1)} \\
0_{1 \times(d+1)} & (c, \mathbf{e})
\end{array}\right), \quad E_{2}=\left(0_{L \times(d+2)}, \quad \mathcal{I}_{L}\right), \quad \mathbf{e}=(1, \ldots, 1) \in \mathbb{R}^{L} .
$$

Here $d \in\{1,2,3\}$ is the spatial dimension of the problem. In (3.4) we have just given the full three-dimensional case but it is clear what the lower dimensional versions are. Conditions (ii) and (iii) have been verified in Proposition 4.1.

To check condition (iv) for (3.4), we compute the Jacobian of $Q=Q(U)$ at $\bar{U}$ :

$$
Q_{U}(\bar{U})=\bar{\rho}\left(\begin{array}{cc}
0_{(d+1) \times(d+2)} & 0_{(d+1) \times L} \\
-L b^{\prime}(\theta) \theta_{\tilde{u}} & \mathbf{e} \\
c b^{\prime}(\theta) \mathbf{e}^{T} \theta_{\tilde{u}} & -c \mathcal{I}_{L}
\end{array}\right)
$$

with $\tilde{u}=\left(\rho, \rho \mathbf{v}^{T}, \rho e\right)$. From this expression of $Q_{U}(\bar{U})$, it is easy to see that

$$
\operatorname{Ker}\left(Q_{U}(\bar{U})\right)=\left\{(\mathbf{f}, \mathbf{g}): \mathbf{f} \in \mathbb{R}^{d+2}, \quad \mathbf{g} \in \mathbb{R}^{L} \quad \text { and } \quad \mathbf{g}=b^{\prime}(\bar{\theta}) \theta_{\tilde{u}}(\bar{U}) \mathbf{f e}^{T}\right\} .
$$

At this point, we state the following simple fact.

Proposition 5.1. If there is a vector $(\mathbf{f}, \mathbf{g}) \in \operatorname{Ker}\left(Q_{U}(\bar{U})\right)$ which is an eigenvector of $A(\boldsymbol{\xi}):=$ $\sum_{j} \xi_{j} F_{j U}(\bar{U})$ with some $\boldsymbol{\xi} \in \mathcal{S}^{d-1}$, then $\mathbf{f}$ is an eigenvector for the classical Euler equations associated with the same eigenvalue.

Proof. Obviously, such an $\mathbf{f}$ must be non-zero. By inspection, $A(\boldsymbol{\xi})$ is block-diagonal with respect to the hydromechanical and radiative unknowns. Hence, $\mathbf{f}$ is an eigenvector for the classical Euler equations associated with the same eigenvalue.

Proposition 5.2. Consider the discrete-ordinate RHD-system (3.4) and assume the rank condition

$$
\operatorname{Rank}\left[\boldsymbol{\omega}_{2}-\boldsymbol{\omega}_{1}, \boldsymbol{\omega}_{3}-\boldsymbol{\omega}_{1}, \cdots, \boldsymbol{\omega}_{L}-\boldsymbol{\omega}_{1}\right]=d
$$

holds true. For $\bar{U} \in \mathcal{U}$ with $Q(\bar{U})=0$, condition (iv) of Theorem 2.1 is satisfied if and only if $d=1$.

Proof. Assume that there is a $\boldsymbol{\xi} \in \mathcal{S}^{d-1}$ such that $(\mathbf{f}, \mathbf{g})=\left(z_{1}, \ldots, z_{d+2}, y_{1}, \ldots, y_{L}\right) \in$ $\operatorname{Ker}\left(Q_{U}(\bar{U})\right)$ is an eigenvector of the characteristic matrix $A(\boldsymbol{\xi}):=\sum_{j} \xi_{j} F_{j U}(\bar{U})$. Let $\lambda=\lambda(\boldsymbol{\xi})$ be the associated eigenvalue. By Proposition 5.1, vector $\mathbf{f} \in \mathbb{R}^{d+2}$ is an eigenvector for the Euler equations associated with the same eigenvalue and $\mathbf{g} \in \mathbb{R}^{L}$ satisfies

$$
c \boldsymbol{\omega}_{l} \cdot \boldsymbol{\xi} y_{l}=\lambda y_{l}
$$

for $l=1, \ldots, L$. By the rank condition, the set $\left\{\boldsymbol{\omega}_{l} \cdot \boldsymbol{\xi} \mid l=1,2, \cdots, L\right\}$ contains at least two distinct numbers and therefore there is an $l$ such that $y_{l}=0$. Due to (5.3), this happens if and only if $\theta_{\tilde{u}}(\bar{U}) \cdot \mathbf{f}=0$, because $b=b(\theta)$ is strictly increasing (cf. (1.6)).

Now we turn to analyse $\theta_{\tilde{u}}(\bar{U}) \cdot \mathbf{f}$. This quantity is invariant under transformations of dependent variables $\tilde{u}$, since $\mathbf{f}$ is an eigenvector for the Euler equation. Thus, we choose the primitive variables $\left(\rho, \mathbf{v}^{T}, \theta\right)$. With the primitive variables, we use the classical relation (1.5) to rewrite the Euler equations as

$$
\begin{aligned}
& \rho_{t}+\mathbf{v} \cdot \nabla \rho+\rho \operatorname{div} \mathbf{v}=0 \\
& \mathbf{v}_{t}+\mathbf{v} \cdot \nabla \mathbf{v}+\nabla p / \rho=0 \\
& \theta_{t}+\mathbf{v} \cdot \nabla \theta+a \operatorname{div} \mathbf{v}=0
\end{aligned}
$$


with $a=\frac{\theta p_{\theta}}{\rho \varepsilon_{\theta}}$. The characteristic matrix is

$$
\tilde{A}(\boldsymbol{\xi})=\mathbf{v} \cdot \boldsymbol{\xi} \mathcal{I}_{(d+2) \times(d+2)}+\left(\begin{array}{ccc}
0 & \rho \boldsymbol{\xi}^{T} & 0 \\
\left(p_{\rho} / \rho\right) \boldsymbol{\xi} & 0_{d \times d} & \left(p_{\theta} / \rho\right) \boldsymbol{\xi} \\
0 & a \boldsymbol{\xi}^{T} & 0
\end{array}\right) .
$$

For $d>1$, the matrix $\tilde{A}$ has three eigenspaces

$$
\left(\begin{array}{c}
\rho \\
-\sqrt{p_{\rho}+a p_{\theta} / \rho} \boldsymbol{\xi} \\
a
\end{array}\right), \quad \operatorname{span}\left\{\left(\begin{array}{c}
p_{\theta} \\
0_{d \times 1} \\
-p_{\rho}
\end{array}\right),\left(\begin{array}{c}
0 \\
\boldsymbol{\xi}^{\perp} \\
0
\end{array}\right)\right\}, \quad\left(\begin{array}{c}
\rho \\
\sqrt{p_{\rho}+a p_{\theta} / \rho} \boldsymbol{\xi} \\
a
\end{array}\right) .
$$

Thus, $\theta_{\tilde{u}}(\bar{U}) \cdot \mathbf{f}$ vanishes if

$$
\mathbf{f}=\left(\begin{array}{c}
0 \\
\boldsymbol{\xi}^{\perp} \\
0
\end{array}\right)
$$

Consequently, condition (iv) is not satisfied in this case.

However, for $d=1,\left(0, \boldsymbol{\xi}^{\perp}, 0\right)^{T}$ is not an eigenvector anymore and $\theta_{\tilde{u}}(\bar{U}) \cdot \mathbf{f}$ can only be

$$
a \text { or }-p_{\rho}
$$

which do not vanish due to the thermodynamical assumption (1.4). Thus, condition (iv) is verified for the one-dimensional case.

The above discussions show that conditions of Theorem 2.1 hold true for the one-dimensional discrete-ordinate systems (3.4). Thus, we have

Theorem 5.3. Consider the one-dimensional discrete-ordinate systems (3.4). Assume the rank condition of Proposition 5.2, let $\bar{U}$ be a constant equilibrium state and $U_{0}: \mathbb{R} \rightarrow \mathcal{U}$ such that $U_{0}-\bar{U} \in H^{s}(\mathbb{R})$ for some integer $s \geq 2$. If $U_{0}-\bar{U}$ is sufficiently small in $H^{s}(\mathbb{R})$, then the Cauchy problem of the one-dimensional system (3.4) with initial data $U_{0}$ has a unique global-in-time classical solution $U$ with $U-\bar{U} \in C\left([0, \infty) ; H^{s}(\mathbb{R})\right)$.

\section{Radiative Profiles for RHD-Shock Waves}

This section is devoted to proving the existence of smooth traveling waves for the discreteordinate RHD-system (3.4), by using Theorem 2.2. Such traveling waves are naturally associated with shock waves for the corresponding equlibrium systems [16, 2]. Their existence is due to the radiation, just as viscous shock profiles are due to viscosity. Therefore, we call them radiative (shock) profiles. The existence also indicates that the shock waves have (radiative) structure.

For the sake of simplicity, we assume that, for the RHD-system (3.4), the fixed equilibrium state $\bar{U}$ in Theorem 2.2 is static, that is, $\overline{\mathbf{v}}=0$. Moreover, we assume that

$$
\sum_{l=1}^{L} \omega_{l}=0
$$

Choose the matrices $E_{1}$ and $E_{2}$ in Theorem 2.1 as in (5.2). Then, under the assumption (6.1), the corresponding equilibrium system (2.4) for the unknown vector $u=(\rho, \rho \mathbf{v}, \rho e+$ 
$\left.c^{-1} L b(\theta)\right)$ is

$$
\begin{aligned}
\rho_{t}+\operatorname{div}(\rho \mathbf{v}) & =0, \\
(\rho \mathbf{v})_{t}+\operatorname{div}(\rho \mathbf{v} \otimes \mathbf{v})+\nabla p & =0, \\
\left(\rho e+c^{-1} L b(\theta)\right)_{t}+\operatorname{div}(\rho e \mathbf{v}+p \mathbf{v}) & =0 .
\end{aligned}
$$

To exploit Theorem 2.2, we analyse the characteristic fields of (6.2) to obtain

Proposition 6.1. (1). For any $\boldsymbol{\xi} \in \mathcal{S}^{d-1}$, the characteristic matrix $\sum_{j=1}^{d} \xi_{j} f_{j u}(\bar{u})$ of the equilibrium system (6.2) has $(d+2)$ real eigenvalues

$$
\lambda_{1}<\lambda_{2}=\ldots=\lambda_{d+1}=\overline{\mathbf{v}} \cdot \boldsymbol{\xi}=0<\lambda_{d+2}
$$

with

$$
\lambda_{1, d+2}=\mp \sqrt{p_{\rho}+\frac{p_{\theta} \beta}{\rho}}, \quad \beta=\beta(\rho, \theta)=\frac{c \theta p_{\theta}}{c \rho \varepsilon_{\theta}+L b^{\prime}(\theta)} .
$$

(2). The first and $(d+2)$-th characteristic fields are both genuinely nonlinear near $\bar{u}$.

Proof. It is known from [14] that these properties of the characteristic fields are invariant under transformations of dependent variables. Thus, we use the classical relation (1.5) to rewrite (6.2) in terms of the primitive variables $(\rho, \mathbf{v}, \theta)$ :

$$
\begin{aligned}
\rho_{t}+\mathbf{v} \cdot \nabla \rho+\rho \operatorname{div} \mathbf{v} & =0, \\
\mathbf{v}_{t}+\mathbf{v} \cdot \nabla \mathbf{v}+\nabla p / \rho & =0, \\
\theta_{t}+(1+\alpha) \mathbf{v} \cdot \nabla \theta+\beta \operatorname{div} \mathbf{v} & =0 .
\end{aligned}
$$

Here

$$
\alpha=\alpha(\rho, \theta)=-\frac{L b^{\prime}(\theta)}{c \rho \varepsilon_{\theta}+L b^{\prime}(\theta)} .
$$

Then the characteristic matrix is similar to

$$
A(\boldsymbol{\xi}):=(\mathbf{v} \cdot \boldsymbol{\xi}) \mathcal{I}_{d+2}+\frac{1}{\rho}\left(\begin{array}{ccc}
0 & \rho^{2} \boldsymbol{\xi}^{T} & 0 \\
p_{\rho} \boldsymbol{\xi} & 0 & p_{\theta} \boldsymbol{\xi} \\
0 & \beta \rho \boldsymbol{\xi}^{T} & \alpha \rho \mathbf{v} \cdot \boldsymbol{\xi}
\end{array}\right) \quad\left(\boldsymbol{\xi} \in \mathcal{S}^{d-1}\right) .
$$

It is obvious that $\mathbf{v} \cdot \boldsymbol{\xi}$ is an eigenvalue of (at least) multiplicity $(d-1)$ with $(d-1)$-dimensional eigenspace $\operatorname{span}\left(\left\{\left(0, \boldsymbol{\xi}^{\perp}, 0\right) \mid \boldsymbol{\xi} \in \mathcal{S}^{d-1}\right\}\right.$. The remaining three eigenvalues are $\lambda+\mathbf{v} \cdot \boldsymbol{\xi}$ with $\lambda$ solving the equation

$$
\rho\left(p_{\rho}-\lambda^{2}\right)(\lambda-\alpha \mathbf{v} \cdot \boldsymbol{\xi})+p_{\theta} \beta \lambda=0 .
$$

The associated eigenvectors are

$$
\left(\rho, \lambda \boldsymbol{\xi}^{T}, \frac{\rho\left(\lambda^{2}-p_{\rho}\right)}{p_{\theta}}\right)^{T} .
$$

Consequently, at the static state $\bar{u}$ where $\overline{\mathbf{v}}=0$, we have $\lambda_{2}=\ldots=\lambda_{d+1}=0$. Note that $\lambda=0$ is a solution to equation (6.6) if $\alpha \mathbf{v} \cdot \boldsymbol{\xi}=0$. Now the equation (6.6) reduces to

$$
\rho \lambda^{2}=\rho p_{\rho}+p_{\theta} \beta>0
$$


for the other two eigenvalues. The positivity is a consequence of (1.4). Thus, the two eigenvalues $\lambda_{1}$ and $\lambda_{d+2}$ are those given in (6.3).

In addition, by using (6.6) it is easy to verify that the two characteristic fields are genuinely nonlinear near $\bar{u}$. This completes the proof.

Next we consider the Rankine-Hugoniot relations for (6.2) with the fixed lefthand state $\bar{u}$ :

$$
\begin{aligned}
\sigma[\rho] & =\boldsymbol{\xi} \cdot[\rho \mathbf{v}], \\
\sigma[\rho \mathbf{v}] & =\boldsymbol{\xi} \cdot[\rho \mathbf{v} \otimes \mathbf{v}+p], \\
\sigma\left[\rho e+c^{-1} L b(\theta)\right] & =\boldsymbol{\xi} \cdot[\rho e \mathbf{v}+v p] .
\end{aligned}
$$

Here $\boldsymbol{\xi}$ is fixed, the brackets denote the jump $[X]=\bar{X}-X, \sigma \in \mathbb{R}$ is the unknown wave speed, and $u \in(0, \infty) \times \mathbb{R}^{d} \times(0, \infty)$ is the unknown righthand state.

Proposition 6.1 and the standard results for hyperbolic conservation laws [14] ensure that for $k=\{1, d+2\}$, there is a number $\varepsilon_{0}>0$, and smooth functions $\sigma_{k}:\left(-\varepsilon_{0}, \varepsilon_{0}\right) \rightarrow \mathbb{R}$ and $u_{k}:\left(-\varepsilon_{0}, \varepsilon_{0}\right) \rightarrow(0, \infty) \times \mathbb{R}^{d} \times(0, \infty)$ such that $(\sigma, u)=\left(\sigma_{k}(\varepsilon), u_{k}(\varepsilon)\right)$ solves $(6.7)$ for all $\varepsilon \in\left(-\varepsilon_{0}, \varepsilon_{0}\right)$. Moreover, $\left(\sigma_{k}(0), \bar{u}_{k}(0)\right)=\left(\lambda_{k}(\bar{u}), \bar{u}\right)$ and the shock inequality

$$
\lambda_{k}(\bar{u})=\sigma_{k}(0)>\sigma_{k}(\varepsilon), \quad \varepsilon \in\left(-\varepsilon_{0}, 0\right) .
$$

holds. In other words, the system of conservation laws (6.2) has two families of shock waves:

$$
u:=u(\mathbf{x}, t)=\left\{\begin{array}{ll}
\bar{u}, & \boldsymbol{\xi} \cdot \mathbf{x}<\sigma_{k}(\varepsilon) t, \\
u_{k}(\varepsilon), & \boldsymbol{\xi} \cdot \mathbf{x}>\sigma_{k}(\varepsilon) t .
\end{array} \quad\left(\varepsilon \in\left(-\varepsilon_{0}, 0\right), \quad k=1, d+2\right) .\right.
$$

Having these preparations, we fix

$$
\bar{U}:=(\bar{\rho}, 0, \ldots, 0, \bar{\rho} \bar{e}, b(\bar{\theta}), \ldots, b(\bar{\theta}))^{T}
$$

and show that for any $\varepsilon<0$ but sufficiently close to 0 , the discrete-ordinate RHD system (3.4) has a traveling wave solution

$$
U(\mathbf{x}, t)=\phi\left(\boldsymbol{\xi} \cdot \mathbf{x}-\sigma_{k}(\varepsilon) t\right)
$$

satisfying $\phi \in C^{1}(\mathbb{R}), \phi(-\infty)=\bar{U}$, and

$$
\phi(+\infty)=\left(\rho_{k}(\varepsilon),\left(\rho_{k} \mathbf{v}_{k}\right)(\varepsilon),\left(\rho_{k} e_{k}\right)(\varepsilon), b\left(\theta_{k}(\varepsilon)\right), \ldots, b\left(\theta_{k}(\varepsilon)\right)\right)^{T}:=U_{k}(\varepsilon) .
$$

We name such a solution as a radiative structure of shock waves (6.8) for (6.2), which connects $\bar{U}$ on the left to $U_{k}(\varepsilon)$ on the right.

The main result of this section can be precisely stated as

Theorem 6.2. Fix $\boldsymbol{\xi} \in \mathcal{S}^{d-1}$ and $\bar{U}$ with $\overline{\mathbf{v}}=0$ as defined in (6.9). Then for each $k \in$ $\{1, d+2\}$ there is a number $\varepsilon_{0}>0$ such that for any $\varepsilon \in\left(-\varepsilon_{0}, 0\right]$, the RHD-system (3.4) has a radiative structure connecting $\bar{U}$ on the left to $U_{k}(\varepsilon)$ on the right.

Proof. We apply Theorem 2.2 together with Remark 2.2. From Proposition 4.1, Proposition 6.1 , and the discussion preceeding Theorem 6.2, it remains to verify the conditions in (b) of Theorem 2.2.

Let $\lambda_{k}(\bar{u})$ be an eigenvalue of the characteristic matrix $\sum_{j} \xi_{j} F_{j U}(\bar{U})$ for the full system (3.4). From Proposition 6.1 we know that $\lambda_{k}(\bar{u}) \notin\left\{0, \pm \sqrt{p_{\rho}+\rho^{-1} a p_{\theta}}\right\}$ due to $a:=\frac{\theta p_{\theta}}{\rho \varepsilon_{\theta}} \neq \beta$. Here we have suppressed the bar over components of $\bar{U}$ for simplicity. On the other hand, because $\overline{\mathbf{v}}=0$ the eigenvalues of $\sum_{j} \xi_{j} F_{j U}(\bar{U})$ are

$$
\Lambda_{1, d+2}(\boldsymbol{\xi})=\mp \sqrt{p_{\rho}+\rho^{-1} a p_{\theta}}, \quad \Lambda_{2}(\boldsymbol{\xi})=\ldots=\Lambda_{d+1}(\boldsymbol{\xi})=0, \quad \Lambda_{l}(\boldsymbol{\xi})=c \boldsymbol{\xi} \cdot \boldsymbol{\omega}_{l-d-2}
$$


for $l=d+3, \ldots, d+2+L$ (See the proof of Proposition 6.1). Thus, $\lambda_{k}(\bar{u})$ can only coincide with one of the eigenvalues $\Lambda_{l}(\boldsymbol{\xi})$ with $l>d+2$, which are all constant. Consequently, the eigenvalues of $\sum_{j=1}^{d} \xi_{j} F_{j U}(U)$ tending to $\lambda_{k}(\bar{u})$ as $U$ goes to $\bar{U}$ are identical for all $U$ close to $\bar{U}$ (see Remark 2.2). Moreover, we see from Proposition 5.1 that

$$
\operatorname{Ker}\left(Q_{U}(\bar{U})\right) \cap \operatorname{Ker}\left(\sum_{j=1}^{d} \xi_{j} F_{j U}(\bar{U})-\lambda_{k}(\bar{u}) \mathcal{I}_{d+2+L}\right)=\{0\} .
$$

Hence the conditions of Theorem 2.2 are verified and the proof is complete.

\section{Radiative Profiles for Slow and Fast RMHD-Shock Waves}

Now we carry out the analysis of the previous section to the more complex RMHD-system (3.2). Namely, we use Theorem 2.2 to show the existence of smooth traveling waves for the discrete-ordinate RMHD-system (3.2).

Let us check the conditions of Theorems 2.2. Condition (i) of Theorem 2.1 is obviously true with $r=L$ and

$$
E_{1}=\left(\begin{array}{cc}
\mathcal{I}_{6} & 0_{6 \times(L+1)} \\
0_{1 \times 6} & (c, \mathbf{e})
\end{array}\right), \quad E_{2}=\left(\begin{array}{cc}
0_{L \times 7}, & \mathcal{I}_{L}
\end{array}\right), \quad \mathbf{e}=(1, \ldots, 1) \in \mathbb{R}^{L} .
$$

Then, under the assumption (6.1), the corresponding equilibrium system for the unknown vector $u=\left(\rho, \rho v, \rho \mathbf{w}, \mathbf{c}, \rho \mathcal{E}+c^{-1} L b(\theta)\right)$ is

$$
\begin{aligned}
\rho_{t}+(\rho v)_{x} & =0, \\
(\rho v)_{t}+\left(\rho v^{2}+p+|\mathbf{c}|^{2} / 2\right)_{x} & =0, \\
(\rho \mathbf{w})_{t}+(\rho v \mathbf{w}-\mathbf{c})_{x} & =0, \\
\mathbf{c}_{t}+(v \mathbf{c}-\mathbf{w})_{x} & =0, \\
\left(\rho \mathcal{E}+c^{-1} L b(\theta)\right)_{t}+\left(\rho \mathcal{E} v+v p+v|\mathbf{c}|^{2} / 2-\mathbf{w} \cdot \mathbf{c}\right)_{x} & =0 .
\end{aligned}
$$

Analogously to Proposition 6.1, we have

Proposition 7.1. Let $\bar{u}=\left(\bar{\rho}, 0, \bar{\rho} \overline{\mathbf{w}}, \overline{\mathbf{c}}, \bar{\rho} \overline{\mathcal{E}}+c^{-1} L b(\bar{\theta})\right) \in(0, \infty) \times\{0\} \times \mathbb{R}^{4} \times(0, \infty)$ with $\overline{\mathbf{c}} \neq 0$. Then the following two statements hold.

(i) The characteristic matrix $f_{j u}(\bar{u})$ of the equilibrium system (7.1) has seven distinct eigenvalues

$$
\lambda_{1}<\lambda_{2}<\ldots<\lambda_{7}
$$

where

$$
\begin{aligned}
& \lambda_{1,7}^{2}=\frac{|\overline{\mathbf{c}}|^{2}+1+\chi+\sqrt{\left(|\overline{\mathbf{c}}|^{2}+1+\chi\right)^{2}-4 \chi}}{2 \rho}, \\
& \lambda_{3,5}^{2}=\frac{|\overline{\mathbf{c}}|^{2}+1+\chi-\sqrt{\left(|\overline{\mathbf{c}}|^{2}+1+\chi\right)^{2}-4 \chi}}{2 \rho}
\end{aligned}
$$

with $\chi=\bar{\rho} p_{\rho}(\bar{\rho}, \bar{\theta})+\beta p_{\theta}(\bar{\rho}, \bar{\theta})$ and $\beta=\beta(\bar{\rho}, \bar{\theta})$ as defined in (6.3).

(ii) The characteristic fields of (7.1) associated to the eigenvalues $\lambda_{k}, k \in\{1,3,5,7\}$, are genuinely nonlinear near $\bar{u}$. 
Proof. In order to analyse the characteristic fields, we rewrite (7.1) in terms of the primitive variables $(\rho, v, \mathbf{w}, \mathbf{c}, \theta)$, as in (6.4), and obtain

$$
\begin{aligned}
\rho_{t}+v \rho_{x}+\rho v_{x} & =0, \\
v_{t}+v v_{x}+p_{x} / \rho+\mathbf{c} \cdot \mathbf{c}_{x} / \rho & =0, \\
\mathbf{w}_{t}+v \mathbf{w}_{x}-\mathbf{c}_{x} / \rho & =0, \\
\mathbf{c}_{t}+v \mathbf{c}_{x}+\mathbf{c} v_{x}-\mathbf{w}_{x} & =0, \\
\theta_{t}+v(1+\alpha) \theta_{x}+\beta v_{x} & =0 .
\end{aligned}
$$

Here $\alpha=\alpha(\rho, \theta)$ and $\beta=\beta(\rho, \theta)$ are defined as in (6.5) and (6.3). The characteristic matrix of system $(7.3)$ is

$$
A(u)=v \mathcal{I}_{7}+\frac{1}{\rho}\left(\begin{array}{ccccccc}
0 & \rho^{2} & 0 & 0 & 0 & 0 & 0 \\
p_{\rho} & 0 & 0 & 0 & c_{1} & c_{2} & p_{\theta} \\
0 & 0 & 0 & 0 & -1 & 0 & 0 \\
0 & 0 & 0 & 0 & 0 & -1 & 0 \\
0 & \rho c_{1} & -\rho & 0 & 0 & 0 & 0 \\
0 & \rho c_{2} & 0 & -\rho & 0 & 0 & 0 \\
0 & \beta \rho & 0 & 0 & 0 & 0 & \alpha \rho v
\end{array}\right)
$$

Here we have written $\mathbf{c}=\left(c_{1}, c_{2}\right)^{T}$.

The eigenvalues of $A(\bar{u})$ can be determined as follows. For simplicity, we suppress the bar over components of $\bar{u}$. By $v=0, A(\bar{u})$ has two eigenvalues $\lambda_{2 / 6}:=\mp 1 / \sqrt{\rho}$ with $\left(0,0, \mathbf{c}^{\perp},-\lambda_{2 / 6} \rho \mathbf{c}^{\perp}, 0\right)^{T}$ as associated eigenvectors (Note that we have assumed $\left.\mathbf{c} \neq 0\right)$. The two characteristic fields are linearly degenerate. The remaining eigenvalues are solutions of the equation

$$
\chi \lambda\left(1-\rho \lambda^{2}\right)-\rho \lambda^{3}|\mathbf{c}|^{2}=\rho\left(1-\rho \lambda^{2}\right) \lambda^{3}
$$

with

$$
\left(\rho, \lambda, \frac{\lambda}{1-\lambda^{2} \rho} \mathbf{c},-\frac{\lambda^{2} \rho}{1-\lambda^{2} \rho} \mathbf{c}, \frac{\beta \lambda}{\lambda-\alpha v}\right)^{T}
$$

as associated eigenvectors. Observe that $\lambda_{4}:=0$ is a solution to (7.4). Thus, the rest four eigenvalues solve the quadratic equation for $\rho \lambda^{2}$ :

$$
\chi\left(1-\rho \lambda^{2}\right)-\rho \lambda^{2}|\mathbf{c}|^{2}=\rho\left(1-\rho \lambda^{2}\right) \lambda^{2},
$$

which can be explicitly solved to obtain

$$
\rho \lambda^{2}=\frac{|\mathbf{c}|^{2}+1+\chi \pm \sqrt{\left(|\mathbf{c}|^{2}+1+\chi\right)^{2}-4 \chi}}{2} .
$$

Since $\mathbf{c} \neq 0$ and $\chi>0$, it is not difficult to see that

$$
0<\frac{|\mathbf{c}|^{2}+1+\chi-\sqrt{\left(|\mathbf{c}|^{2}+1+\chi\right)^{2}-4 \chi}}{2}<1<\frac{|\mathbf{c}|^{2}+1+\chi+\sqrt{\left(|\mathbf{c}|^{2}+1+\chi\right)^{2}-4 \chi}}{2} .
$$

Note that $1=\rho \lambda_{2 / 6}^{2}$. Therefore, $A(\bar{u})$ has seven distinct eigenvalues. We order the last four eigenvalues by size and denote them by $\lambda_{k}(\bar{u})$ with $k \in\{1,3,5,7\}$.

In addition, by using (7.4) it is a tedious but straightforward computation to verify that the last four characteristic fields are genuinely nonlinear near $v=0$ and $\mathbf{c} \neq 0$. 
Now fix some static state $\bar{u} \in(0, \infty) \times\{0\} \times \mathbb{R}^{4} \times(0, \infty)$ with $\overline{\mathbf{c}} \neq 0$ as in Proposition 7.1. With the same notation and arguments as in the previous section, we get four families (according to the four genuinely nonlinear fields) of shock waves for the equilibrium system (7.1):

$$
u:=u(x, t)=\left\{\begin{array}{ll}
\bar{u}, & x<\sigma_{k}(\varepsilon) t, \\
u_{k}(\varepsilon), & x>\sigma_{k}(\varepsilon) t .
\end{array} \quad\left(\varepsilon \in\left(-\varepsilon_{0}, 0\right), \quad k=1,3,5,7\right) .\right.
$$

Having these preparations, we fix

$$
\bar{U}:=(\bar{\rho}, 0, \bar{\rho} \overline{\mathbf{w}}, \overline{\mathbf{c}}, \bar{\rho} \overline{\mathcal{E}}, b(\bar{\theta}), \ldots, b(\bar{\theta}))^{T},
$$

with $\overline{\mathbf{c}} \neq 0$, and show that for any $\varepsilon<0$ but sufficiently close to 0 , the discrete-ordinate RMHD system (3.2) has a traveling wave solution

$$
U(x, t)=\phi\left(x-\sigma_{k}(\varepsilon) t\right)
$$

satisfying $\phi \in C^{1}(\mathbb{R}), \phi(-\infty)=\bar{U}$, and

$$
\phi(+\infty)=\left(\rho_{k}(\varepsilon),\left(\rho_{k} v_{k}\right)(\varepsilon),\left(\rho_{k} \mathbf{w}_{k}\right)(\varepsilon), \mathbf{c}_{k}(\varepsilon), b\left(\theta_{k}(\varepsilon)\right), \ldots, b\left(\theta_{k}(\varepsilon)\right)\right)^{T}=: U_{k}(\varepsilon) .
$$

The last main result of this paper is

Theorem 7.2. Fix $\bar{U}$ as defined in (7.5). Then for each $k \in\{1,3,5,7\}$ there is a number $\varepsilon_{0}>0$ such that for all $\varepsilon \in\left(-\varepsilon_{0}, 0\right]$, the RMHD-system (3.2) has a radiative structure connecting $\bar{U}$ on the left and $U_{k}(\varepsilon)$ on the right.

Proof. We again apply Theorem 2.2 together with Remark 2.2. The conditions (i)-(iii) have been verified in the beginning of this section and in Proposition 4.2. For the other conditions, we refer to Proposition 7.1 and the discussions preceeding Theorem 7.2. Thus, it remains to verify the conditions in (b) of Theorem 2.2. To this end, we refer to the proof of Proposition 7.1 and see that eigenvalues of the characteristic matrix $F_{1 U}(\bar{U})$ are

$$
\begin{aligned}
\Lambda_{1,7}^{2} & =\frac{|\mathbf{c}|^{2}+1+\chi_{0}+\sqrt{\left(|\mathbf{c}|^{2}+1+\chi_{0}\right)^{2}-4 \chi_{0}}}{2 \rho} \\
\Lambda_{3,5}^{2} & =\frac{|\mathbf{c}|^{2}+1+\chi_{0}-\sqrt{\left(|\mathbf{c}|^{2}+1+\chi_{0}\right)^{2}-4 \chi_{0}}}{2 \rho} \\
\Lambda_{2 / 6}^{2} & =\frac{1}{\rho} \\
\Lambda_{4} & =0 \\
\Lambda_{l} & =c \omega_{l-7}
\end{aligned}
$$

for $l=8, \ldots, L+7$, where $\chi_{0}=\rho p_{\rho}+\frac{\theta p_{\theta}^{2}}{\rho \varepsilon_{\theta}}$. Here we have suppressed the bar over components of $\bar{U}$ for simplicity.

Next let $\lambda_{k}(\bar{u})$ be an eigenvalue of the $F_{1 U}(\bar{U})$. Since $\chi \neq \chi_{0}$, we know from Proposition 7.1 that $\lambda_{k}(\bar{u}) \notin\left\{\Lambda_{k}: k=1,2, \cdots, 7\right\}$. Thus, $\lambda_{k}(\bar{u})$ can only coincide with one of the eigenvalues $\Lambda_{l}$ with $l>7$, which are all constant. Consequently, the eigenvalues of $F_{1 U}(U)$ tending to $\lambda_{k}(\bar{u})$ as $U$ goes to $\bar{U}$ are identical for all $U$ close to $\bar{U}$ (see Remark 2.2). Moreover, it is easy to see, as in Proposition 5.1, that if $\operatorname{Ker}\left(Q_{U}(\bar{U})\right) \cap \operatorname{Ker}\left(F_{1 U}(\bar{U})-\lambda_{k}(\bar{u}) \mathcal{I}_{L+7}\right)$ contains a non-zero vector, then $\lambda_{k}(\bar{u}) \in\left\{\Lambda_{k}: k=1,2, \cdots, 7\right\}$. Hence the conditions of Theorem 2.2 are verified and the proof is complete. 


\section{REFERENCES}

[1] A. Dedner \& D. Kröner \& C. Rohde \& M. Wesenberg, Radiation-Magnetohydrodynamics: Analysis for Model Problems and 3D-Simulations for the Full System, In: G. Warnecke (Ed.), Analysis und Numerik für Erhaltungssätze, Springer (2005).

[2] A. Dressel \& W.-A. Yong, Existence of traveling wave solutions for hyperbolic systems of balance laws, Arch. Ration. Mech. Anal. 182 (2006), 49-75.

[3] E. Godlewski \& P.-A. Raviart, Hyperbolic systems of conservation laws, Mathématiques \& Applications 3-4. Ellipses, Paris (1991).

[4] S. R. de Groot \& P. Mazur, Non-Equilibrium Thermodynamics, North-Holland Publishing Company, Amsterdam, 1962.

[5] M.A. Heaslet \& B.S. Baldwin, Predictions of the structure of radiation-resisted shock waves, Phys. Fluids 6 (1963), 781-791.

[6] S. Kawashima \& Y.Nikkuni \& S. Nishibata, The initial value problem for hyperbolic-elliptic coupled systems and applications to radiation hydrodynamics, H. Freistühler(ed.): Analysis of systems of conservation laws, Chapman\&Hall monographs and surveys in pure and applied mathematics 99, 87-127 (1998).

[7] C. Lattanzio \& C. Mascia \& D. Serre, Shock waves for radiative hyperbolic-elliptic systems, Indiana Univ. Math. J., to appear.

[8] H. Lin \& J.F. Coulombel \& T. Goudon, Shock profiles for non-equilibrium radiating gases. Phys. D 218, no. 1, 83-94 (2006).

[9] D. Mihalas \& B. Mihalas, Foundations of radiation hydrodynamics, Oxford University Press, New York, 1984.

[10] P. Leopardi, A partition of the unit sphere into regions of equal area and small diameter. Electron. Trans. Numer. Anal. 25 (2006), 309-327.

[11] G. C. Pomraning, The equations of radiation hydrodynamics, Pergamon Press, Oxford, New York (1973).

[12] C. Rohde \& W. Zajaczkowski, On the Cauchy problem for the equations of ideal compressible MHD fluids with radiation. Appl. Math. 48, no. 4 (2003), 257-277.

[13] Y. Shizuta \& S. Kawashima, Systems of equations of hyperbolic-parabolic type with applications to the discrete Boltzmann equation. Hokkaido Math. J. 14 (1985), 249-275.

[14] J. Smoller,Shock waves and reaction-diffusion equations, Springer, New York, 1994.

[15] W.-A. Yong, Entropy and global existence for hyperbolic balance laws, Arch. Rat. Mech. Anal. 172 (2004), 247-266.

[16] W.-A. Yong \& K. Zumbrun, Existence of relaxation shock profiles for hyperbolic conservation laws, Siam J. Appl. Math. 60 (2000), 1565-1575.

[17] X. Zhong \& S. Jiang, Local Existence and Finite-time Blow-up in Multidimensional Radiation Hydrodynamics, J. Math. Fluid Dynamics, DOI 10.1007/s00021-005-0213-3.

Institut für Angewandte Analysis und Numerische Simulation, Universität Stuttgart, 70569 Stuttgart, Germany

E-mail address: crohde@mathematik.uni-stuttgart.de

Zhou Pei-Yuan Center for Appl. Math., Tsinghua University, Beijing 100084, China

E-mail address: wayong@mail.tsinghua.edu.cn 